\title{
Cutting a Polytope
}

\author{
WILLIAM JOCKUSCH ${ }^{1}$ and NAGABHUSHANA PRABHU ${ }^{2}$ \\ ${ }^{1}$ University of Michigan, Ann Arbor, MI 48109, U.S.A. \\ ${ }^{2}$ Purdue University, West Lafayette, IN 47907, U.S.A.
}

(Received: 23 November 1993)

\begin{abstract}
We investigate whether it is the case that for every convex $d$-polytope $P$ and pair of distinct vertices $x$ and $y$ of $P$, there exists a hyperplane passing through $x$ and $y$ which cuts $P$ into two smaller $d$-polytopes, one of which has fewer facets than $P$. Such a result would lead to inductive proofs of Conjectures 1 and 2 below. However, for $d \geq 4$, our answer is in the negative.
\end{abstract}

Mathematics Subject Classifications (1991): Primary 52; Secondary 05.

\section{Introduction}

Several unresolved problems about convex polytopes concern proving the existence of certain paths between two prespecified vertices of the polytope. Below, we cite three outstanding conjectures as examples.

DEFINITION 1. A $k$-path on a $d$-polytope $(k<d)$ between vertices $x$ and $y$ is a sequence of $k$-faces, where two consecutive $k$-faces in the sequence share a $(k-1)$-face, $x$ belongs to the first $k$-face in the sequence and $y$ to the last.

CONJECTURE 1 ([11]). Let $d=d_{1}+d_{2}+\cdots+d_{m}$ be a partition of the number $d$. Then, between any pair of vertices $v, w$ of a d-polytope $P$, there exist a $d_{1}$-path, $a d_{2}$-path, $\ldots$, and a $d_{m}$-path where all the $m$ paths on $P$ are pairwise disjoint except for the end vertices $v, w$.

The conjecture is unresolved even for the case $d=(d-1)+1$. When $d_{1}=$ $d_{2}=\cdots=d_{d}=1$ the above conjecture reduces to Balinski's theorem [1]. The conjecture is true when the vertices $v$ and $w$ are adjacent from which it follows that it is true for all neighborly polytopes [15].

CONJECTURE 2 ([4]). Between any two vertices of a d-polytope there exist $\left(\begin{array}{l}d \\ k\end{array}\right)$ $k$-paths such that no two of the $k$-paths have one or more $k$-faces in common.

CONJECTURE 3. Given a d-polytope $P$ and $a$ pair of its vertices $x$ and $y$, there exists a refinement homeomorphism [5] from $P$ to the $d$-simplex $T^{d}$, which maps $x$ and $y$ onto two vertices of $T^{d}$.

Micha A. Perles suggested that Conjecture 3 would imply both Conjectures 1 and 2. Grünbaum's proof of Grünbaum-Motzkin theorem [6] also proves a variant of 
Conjecture 3 in which we insist that one prespecified vertex of $P$ (instead of two) be mapped into a vertex of a $d$-simplex.

A fourth example of an unresolved conjecture in which two prespecified vertices of a $d$-polytope play a special role concerns a generalization of Grünbaum-Motzkin theorem. See [12] for the statement of and partial results concerning the conjecture.

In all of the above problems we are required to prove the existence of certain paths between two special prespecified vertices of the polytope.

If for every pair of vertices $x$ and $y$ of a polytope one could find a hyperplane $H$ such that

(1) $H$ contains $x$ and $y$ and

(2) $H$ cuts the $d$-polytope $P$ into two smaller $d$-polytopes $P_{1}$ and $P_{2}$ with $P_{1}$ having fewer facets than $P$

then one could resolve Conjectures 1 and 2 using induction on the number of facets in a $d$-polytope (the conjectures are trivially true for a $d$-simplex, the $d$-polytope with minimum number of facets).

Hence our interest was to prove that for every d-polytope $P$ (except a d-simplex) and for every pair of vertices $x$ and $y$ in $P$, one can cut $P$ into two smaller $d$ polytopes, using a hyperplane containing $x$ and $y$, such that one of the smaller polytopes has fewer facets than $P$.

However, it turns out that there are curious 4-dimensional polytopes that do not admit the cuts described in the previous paragraph (see Theorem 2). The result seems rather surprising - partly due to the fact that every 3-dimensional polytope (except 3-simplex) does admit a cut as described in the previous paragraph (see Theorem 3).

Our main result - Theorem 2 - contributes to the growing understanding of how hyperplanes intersect the boundary of polytopes. We mention below a few examples of results about intersection of hyperplanes with the boundary of a polytope. Bezdek, Bisztriczky and Connelly [2] showed that a hyperplane can intersect the relative interiors of all the $j$-dimensional faces of a $d$-polytope only if $j \geq\lfloor d / 2\rfloor$. Perles [14] and Prabhu [15] prove stronger version of the above result: if a $k$-flat intersects the relative interiors of all the $j$-faces of a d-polytope then $k \geq 2(d-j)$. Further, Perles and Prabhu show how to construct polytopes with arbitrarily large numbers of vertices in which all the $j$-faces, $j \geq\lfloor d / 2\rfloor$, can be intersected by a $2(d-j)$-flat. We make a special mention of Khovanskii's results [8]. He showed that if a hyperplane $H$ intersects a $d$-polytope $P$, (and $H^{+}$and $H^{-}$ are the two closed halfspaces of $H$ ) then there exist two faces of $P, F_{1} \in H^{+}$and $F_{2} \in H^{-}$such that $\operatorname{dim}\left(F_{1}\right)+\operatorname{dim}\left(F_{2}\right) \geq d-1$. For other results see $[9,10,16]$.

Besides the main result (Theorem 2), Theorem 1 below (that we use to prove Theorem 2) will likely be of independent interest to researchers in Computational Geometry and Polytope Theory.

In Section 2 we discuss and prove Theorem 1 about an arrangement of tetrahedra around the origin. In Section 3, we prove the main result (Theorem 2). Section 4, 
shows that for any two vertices of a 3-polytope $P$ one can find a plane containing the vertices, that cuts the 3-polytope into two smaller 3-polytopes, such that one of the smaller polytopes has fewer facets that $P$; thus 4-polytopes provide the lowest dimensional examples of polytopes that do not admit such cuts.

\section{Arrangement of Tetrahedra}

Let $T$ and $T^{\prime}$ be two tetrahedra positioned such that the origin is in the interior of both. Given such an arrangement can one find a plane $H$ through the origin, that has two of the eight boundary triangles on one side (in a closed halfspace)? For many of the simple arrangements (e.g. $T^{\prime}=-T$ ) it is easily seen that such a plane exists. We show below how to construct and position two tetrahedra such that no plane through the origin can have more than one of the eight boundary triangles on each side.

Two of the eight boundary triangles lie on one side of a plane through the origin if and only if six of the eight vertices do. Hence we have

THEOREM 1. There exist tetrahedra $T$ and $T^{\prime}$ which can be positioned such that

(1) the origin is in the interior of both the tetrahedra; and

(2) no closed half-space whose boundary plane passes through the origin contains more than five of the eight vertices of the two tetrahedra.

Proof. For each nonzero vector $v \in R^{3}$ define a closed dual halfspace

$$
D(v)=\left\{x \in R^{3} \mid\langle v, x\rangle \geq 0\right\} .
$$

Conversely for every closed halfspace $X$ whose boundary contains the origin we can find a dual vector $D^{-1}(X) .\left(D^{-1}(X)\right.$ is not unique; for any $\alpha>0, \alpha D^{-1}(X)$ is also a dual vector of $X$. When we say $D^{-1}(X)$ we mean some nonzero $v$ such that $D(v)=X$.)

Observe that if $p, q$ are two vectors in $R^{3}$ then $p \in D(q)$ if and only if $q \in D(p)$. Let $\operatorname{vert}(T)=\left\{v_{1}, \ldots, v_{4}\right\}$, $\operatorname{vert}\left(T^{\prime}\right)=\left\{v_{5}, \ldots, v_{8}\right\}$ and $V=\operatorname{vert}(T) \cup \operatorname{vert}\left(T^{\prime}\right)$. If six or more of the eight vertices in $V$ are contained in some halfspace $H$ that's bounded by a hyperplane through the origin, then $D^{-1}(H)$ must be contained in the dual halfspaces corresponding to those vertices (i.e. in at least six of the eight halfspaces $\left.D\left(v_{1}\right), \ldots, D\left(v_{8}\right)\right)$. Now we construct a set of eight closed halfspaces $D_{1}, \ldots, D_{8}$ having the following properties:

(1) Dual vectors of $D_{1}, \ldots, D_{4}$ (resp. $D_{5}, \ldots, D_{8}$ ) are the vertices of a tetrahedron $T$ (resp. $T^{\prime}$ ) which contains the origin in its interior.

(2) The intersection of any six of the eight halfspaces $D_{1}, \ldots, D_{8}$ is empty.

Then considering $T$ (resp. $T^{\prime}$ ) to be the convex hull of the dual vectors of $D_{1}, \ldots, D_{4}$ (resp. $D_{5}, \ldots, D_{8}$ ) completes the proof. 
It's convenient to map the problem of constructing $D_{1}, \ldots, D_{8}$, onto the unit 2sphere $S^{2}=\left\{x \in R^{3} \mid\|x\|=1\right\}$. A circle on $S^{2}$ of unit radius is called a great circle and a semicircle of a great circle, a great semicircle. The intersection of $S^{2}$ with a hyperplane through the origin is a great circle. Let $H$ and $H^{\prime}$ be two closed halfspaces bounded by hyperplanes through the origin, such that $h=D^{-1}(H)$ and $h^{\prime}=D^{-1}\left(H^{\prime}\right)$ are linearly independent. Then $S^{2} \cap H \cap H^{\prime}$ will be called a crescent (whose boundary on $S^{2}$ is the union of two great semicircles).

LEMMA 1. Let $H_{1}, \ldots, H_{4}$ be closed halfspaces bounded by hyperplanes through the origin. Further, let $\mathrm{H}_{1} \cap \mathrm{H}_{2} \cap S^{2}$ and $\mathrm{H}_{3} \cap \mathrm{H}_{4} \cap S^{2}$ form crescents $C$ and $C^{\prime}$ that do not intersect. Then $D^{-1}\left(H_{1}\right), \ldots, D^{-1}\left(H_{4}\right)$ are the vertices of a tetrahedron containing the origin in its interior.

Proof. Four vectors $p_{1}, \ldots, p_{4} \in R^{3}$ form the vertices of a tetrahedron containing the origin in its interior if and only if for each nonzero vector $v \in R^{3},\left\langle v, p_{i}\right\rangle>$ 0 for some $1 \leq i \leq 4$.

Let $h_{i}=\bar{D}^{-1}\left(H_{i}\right), i=1, \ldots, 4 .\left\langle v, h_{i}\right\rangle>0$ if and only if $v$ lies in the open halfspace of $H_{i}$. The union of the open halfspaces of $H_{1}$ and $H_{2}$ contains all of $S^{2}$ except the image of $C$ under inversion $I: x \rightarrow-x$. Since $C \cap C^{\prime}=\emptyset$, the open halfspaces corresponding to $H_{1}, \ldots, H_{4}$ cover $S^{2}$ and hence $h_{1}, \ldots, h_{4}$ are the vertices of a tetrahedron containing the origin in its interior.

Thus every pair of nonintersecting crescents on $S^{2}$ corresponds to a tetrahedron with the origin in its interior. We now show that one can draw an arbitrarily large number of mutually nonintersecting crescents on $S^{2}$.

LEMMA 2. There are infinitely many mutually nonintersecting great semicircles on $S^{2}$.

Proof. Call two antipodes of $S^{2}$ the north and south poles. Consider two latitudes $L_{1}$ and $L_{2}$ symmetric about the equator. There is a great circle $C$ on $S^{2}$ tangent to both $L_{1}$ and $L_{2}$. Let $C_{1 / 2}$ be the great semicircle of $C$, with endpoints on $L_{1}$ and $L_{2}$. As $L_{1}$ and $L_{2}$ are rotated in step about the axis determined by the north and south poles, $C_{1 / 2}$ generates a continuum of mutually nonintersecting great semicircles.

PROOF OF THEOREM 1. Consider four mutually nonintersecting great semicircles $G_{1}, \ldots, G_{4}$ on $S^{2}$. They can be widened to form four mutually nonintersecting crescents $C_{1}, \ldots, C_{4}$. Let $D_{1}, D_{2}$ be the halfspaces forming crescent $C_{1}, \ldots, D_{7}, D_{8}$ the halfspaces forming $C_{4}$. If any nonzero vector is contained in six or more of $D_{1}, \ldots, D_{8}$ then it must be contained in at least two of the four nonintersecting crescents, which is impossible. Thus crescents $\left\{C_{1}, C_{2}\right\}$ give us the tetrahedron $T$ and $\left\{C_{3}, C_{4}\right\}$ the tetrahedron $T^{\prime}$. 


\section{Construction of 4-Polytope}

Let $H$ be a hyperplane that cuts a $d$-polytope $P$ into two smaller $d$-polytopes $P_{1}$ and $P_{2} . P_{1}$ has fewer facets than $P$ if and only if two of the facets of $P$ are contained in the closed halfspace of $H$ containing $P_{2}$.

Using the above observation and Theorem 1 we prove

THEOREM 2. There is a convex 4-polytope $P \subset R^{4}$ with vertices $v$ and $w$ for which no hyperplane containing $v$ and $w$ has more than one facet of $P$ in either closed half-space.

Proof. Let $T$ and $T^{\prime}$ be the tetrahedra of Theorem 1 lying in $R^{3}$. Coordinatize $R^{4}$ with $x, y, z$ and $t$ axes and identify $R^{3}$ with the hyperplane $t=0$. Translate $T$ along the $t$-axis to the hyperplane $t=1$ and similarly $T^{\prime}$ to the hyperplane $t=-1$. Let $\varepsilon$ be small enough that every line containing a point of $T^{\prime}$ and the point $v=(0,0,0,1+\varepsilon)$ intersects the interior of $T$, and every line containing a point of $T$ and the point $w=(0,0,0,-1-\varepsilon)$ intersects the interior of $T^{\prime}$. Let $C$ be the cone with vertex $v$ and cross-section $T ; C^{\prime}$ is the cone with vertex $w$ and cross-section $T^{\prime}$; then $P=C \cap C^{\prime}$ is a convex 4-polytope with eight facets - four in the star of $v$ and the other four in the star of $w$ [3]. If possible, let $H$ be a hyperplane containing $\{v, w\}$, that has two facets of $P$ on one side. Since $H$ intersects the interior of $T$ two facets from the star of $v$ cannot lie on the same side of $H$ (and likewise for $w$ ). However if the stars of $v$ and $w$ contribute one facet each then the orthogonal projection of $H$ onto the hyperplane $t=0$ would be a plane in $R^{3}$ passing through the origin and having two of the eight boundary triangles of $T$ and $T^{\prime}$ in one closed halfspace (w.r.t. $R^{3}$ ). Hence $H$ cannot exist.

\section{Cutting 3-Polytopes}

THEOREM 3. Let $P \subset R^{3}$ be any convex 3-polytope; let $v$ and $w$ be two vertices of $P$. Then there is a plane $H$ containing $v$ and $w$ such that at least two facets of $P$ lie in one of the closed halfspaces of $H$.

Proof. If $v$ and $w$ lie on the same facet of $P$, the theorem is clear. Otherwise, let $G$ be a plane normal to the segment $[v, w]$. Let $f_{v}$ and $f_{w}$ be vertex-figures of $v$ and $w$; they can be projected orthogonally onto $G$ to give polygons $\bar{f}_{v}$ and $\bar{f}_{w}$. Clearly the point $\mathbf{0}=[v, w] \cap G$ lies in the relative interiors of both $\bar{f}_{v}$ and $\bar{f}_{w}$. Let $u$ be any vertex of $\bar{f}_{v}$; the line $L=\overleftrightarrow{\mathbf{0 u} u}$ intersects the relative interior of at most one side of $\bar{f}_{v}$ and at most two sides of $\bar{f}_{w}$; hence at least three sides of the two polygons are contained entirely in the closed halfplanes of $L$. So three facets of $P$ are contained entirely in the two closed halfspaces of the plane $H=\operatorname{aff}(u, v, w)$; two of them must lie on the same side of $H$. 


\section{References}

1. Balinski, M. L.: On the graph structure of convex polyhedra in $n$-space, Pacific J. Math. 11 (1961), 431-434.

2. Bezdek, K., Bisztriczky, T. and Connelly, R.: On hyperplanes and polytopes, Monats. Math. 109 (1990), 39-48.

3. Brondsted, A.: An Introduction to Convex Polytopes, Springer-Verlag, 1983.

4. Problems Presentedat the DIMACS Workshopon Polytopes and Convex Sets, Rutgers University, New Brunswick; 8-12 January 1990.

5. Grünbaum, B.: Convex Polytopes, Wiley Interscience, 1966.

6. Grünbaum, B. and Motzkin, T. S.: On polyhedral graphs, in V. Klee (ed.), Convexity, Amer. Math. Soc. Proc. Symp. Pure Math. Vol 7, 1963, pp. 285-290.

7. Emamy-Khansary, M. R.: On the cut-number of the 5-cube, Proc. the Twentieth Southeastern Conf. on Combinatorics, Graph Theory and Computing (Boca Raton, FL, 1989); Congr. Numer. 72 (1990), 179-186.

8. Khovanskii, A. G.: Hyperplane sections of polytopes, toric varieties and discrete groups in Lobachevskii space, Funktsional Anal. Prilozhen 20 (1986), 50-61.

9. Kincses, J.: Convex hull representation of cut polytopes, Preprint, 1988.

10. Kleinschmidt, P. and Pachner, U.: Shadow-boundaries and cuts of convex polytopes, Mathematika 27 (1980), 58-63.

11. Lockeberg, E. R.: in J. Tolke and J. M. Wills (eds), Contributions to Geometry, Proc. of the Geometry Symposium in Siegen, Problem 57, p. 269, 1978.

12. McMullen, P., Gallivan, S. and Lockeberg, E. R.: Complete subgraphs of the graphs of convex polytopes, Discrete Math. 34 (1981), 25-29.

13. O'Neil, P. E.: Hyperplane cuts of an $n$-cube, Discrete Math. 1 (2) (1971), 193-195.

14. Perles, M. A.: Personal communication.

15. Prabhu, N.: Properties of convex polytopes, Ph.D. Dissertation, New York University, 1991.

16. Shephard, G. C.: Sections and projections of convex polytopes, Mathematika 19 (1972), 144-162. 